NOTICE: this is the author's version of a work that was accepted for publication in Systems \& Control Letters. Changes resulting from the publishing process, such as peer review, editing, corrections, structural formatting, and other quality control mechanisms may not be reflected in this document. Changes may have been made to this work since it was submitted for publication. A definitive version was subsequently published in Systems \& Control Letters [VOL58, ISSUE7, 2010] http://dx.doi.org/ 10.1016/j.sysconle.2009.02.006 


\title{
LQ optimal control for 2-D Roesser models of finite extent
}

\author{
Lorenzo Ntogramatzidis ${ }^{\dagger}$ and Michael Cantoni ${ }^{\ddagger}$ \\ $\dagger$ Department of Mathematics and Statistics, Curtin University of Technology, \\ Perth,WA6845, Australia.E-mail: l.ntogramatzidis@curtin.edu.au \\ ${ }^{\ddagger}$ Department of Electrical and Electronic Engineering, The University of \\ Melbourne, Parkville, VIC 3010, Australia.E-mail: cantoni@unimelb.edu.au
}

\begin{abstract}
This paper investigates several aspects of linear-quadratic (LQ) optimal control for Roesser models over a two-dimensional (2-D) signal-index set of finite extent. First, we consider the characterisation and computation of open-loop control laws when constraints on the system semi-states are imposed at both the south-west and north-east boundaries of the frame (i.e. signal-index set) of interest; by virtue of the quarter-plane causal structure of the Roesser model, the south-west and north-east boundary conditions are analogous to initial conditions and terminal constraints, respectively. A necessary and sufficient characterisation of optimality is obtained and explicitly computable formulae are derived to characterise the corresponding control inputs and performance index under reasonable assumptions on the problem data. In the second part of the paper, the problem of optimal LQ control via semistate feedback is considered. A 2-D Riccati-like difference equation is introduced to characterise, in a sufficient sense, a solution to this problem.
\end{abstract}

Key words: Roesser models, linear quadratic optimal control, semi-state feedback.

\section{Introduction}

Two-dimensional (2-D) systems arise naturally in the modelling of processes where the signals of interest are defined over a purely spatial, or mixed spatiotemporal, index set. Standard latent variable (semi-state) models for 2-D systems include the so-called Roesser and Fornasini-Marchesini models; see (Roesser,

\footnotetext{
^ This work was supported in part by the Australian Research Council (DP0664789 and DP0986577).
} 
1975) and (Fornasini and Marchesini, 1978), respectively. A feature of these models is a quarter-plane causal structure, given semi-state constraints on the south-west boundary of the signal-index set, or frame, of interest; such constraints are analogous to initial conditions in a one-dimensional (1-D) setting. These two models are also known to be equivalent in terms of the input-output behaviours they can represent (Kaczorek, 1985).

Optimal control problems for linear Fornasini-Marchesini models are addressed in (Bisiacco and Fornasini, 1990) and (Bisiacco, 1995) over an infinite frame of interest. The optimal control of 2-D systems over a finite frame, on the other hand, is studied for a non-linear form of the Roesser model in ( $\mathrm{Li}$ and Fadali, 1991), where a variational approach is used to obtain necessary conditions for optimality. In this paper, we first consider a linear version of the optimal control problem for Roesser models, with a quadratic measure of performance and affine constraints on the north-east semi-states; these additional constraints are analogous to terminal state constraints as studied in (Ferrante and Ntogramatzidis, 2005) for 1-D systems. For this problem, the corresponding conditions for optimality are shown to be both necessary and sufficient. While this is not surprising in view of the convex nature of the problem, solving the equations that characterise optimality is not straightforward in general. Conditions under which the equations can be explicitly solved to yield the optimal open-loop controls are explored and we provide an expression for the optimal cost.

Unlike the corresponding 1-D problem, it is not always possible to express the 2-D linear-quadratic (LQ) optimal control in terms of a state feedback; see (Bisiacco and Fornasini, 1990) and (Bisiacco, 1995) for further discussion of this point within the context of Fornasini-Marchesini models. Bearing this in mind, the second part of the paper is concerned with the very difficult problem of optimal LQ regulation via static semi-state feedback. We employ a least-squares approach to derive a 2-D Riccati-like difference equation, and corresponding boundary conditions, which together characterise an optimal semi-state feedback gain in the absence of the north-east boundary conditions. It is not known how to handle such condition when a static semi-state feedback is required.

Notation. The symbol $\mathbb{N}$ denotes the natural numbers (including 0 ) and $[X, Y]$ denotes the interval of $\mathbb{N}$ between $X$ and $Y$, inclusive. The Moore-Penrose pseudo-inverse of a matrix $M$ is denoted $M^{\dagger}$ and its transpose by $M^{\top}$.

\section{Problem Statement}

Consider the shift-invariant system of linear (partial) difference equations 


$$
\left[\begin{array}{c}
h_{i+1, j} \\
v_{i, j+1}
\end{array}\right]=A\left[\begin{array}{c}
h_{i, j} \\
v_{i, j}
\end{array}\right]+B u_{i, j}
$$

where $h_{i, j} \in \mathbb{R}^{n_{h}}$ is called the horizontal semi-state, $v_{i, j} \in \mathbb{R}^{n_{v}}$ the vertical semistate and $u_{i, j} \in \mathbb{R}^{m}$ is the control input, all at the signal-index $(i, j)$ in a $2-\mathrm{D}$ frame of interest. This is the so-called Roesser model (Roesser, 1975). In the following, it becomes convenient to partition the matrices $A \in \mathbb{R}^{\left(n_{h}+n_{v}\right) \times\left(n_{h}+n_{v}\right)}$ and $B \in \mathbb{R}^{\left(n_{h}+n_{v}\right) \times m}$ conformably with the semi-state vectors $h$ and $v$; specifically,

$$
A=\left[\begin{array}{cc}
A_{1,1} & A_{1,2} \\
A_{2,1} & A_{2,2}
\end{array}\right] \text { and } B=\left[\begin{array}{c}
B_{1} \\
B_{2}
\end{array}\right]
$$

Given a frame of interest $\mathcal{Q}:=[0, N] \times[0, M]$, the control input $u$ over $\mathcal{Q}$, and south-west boundary conditions

$$
h_{0, j}=a_{j} \in \mathbb{R}^{n_{h}} \text { for } j \in[0, M] \quad \text { and } \quad v_{i, 0}=b_{i} \in \mathbb{R}^{n_{v}} \text { for } i \in[0, N] \text {, }
$$

the system of equations (1) uniquely determines the horizontal semi-state $h$ over the finite frame $\mathcal{H}:=[0, N+1] \times[0, M]$ and the vertical semi-state $v$ over $\mathcal{V}:=[0, N] \times[0, M+1]$. Moreover, the values of the semi-states bear a quarter-plane causal relationship to the control input and one can think of these as evolving towards the north-east boundary of $\mathcal{Q}$; in particular, $h_{i, j}$ and $v_{i, j}$ only depend on $u_{k, l}$ for $(k, l) \in[0, i] \times[0, j] \backslash\{(i, j)\}$.

Given south-west boundary conditions (3), the finite-frame LQ optimal control problem is to construct an input $u$ for $(1)$, over $\mathcal{Q}$, so as to minimise the corresponding quadratic cost

$$
J:=\mathbf{h}_{N+1}^{\top} H \mathbf{h}_{N+1}+\mathbf{v}_{M+1}^{\top} V \mathbf{v}_{M+1}+\sum_{(i, j) \in \mathcal{Q}}\left[h_{i, j}^{\top} v_{i, j}^{\top} u_{i, j}^{\top}\right] \Pi\left[\begin{array}{c}
h_{i, j} \\
v_{i, j} \\
u_{i, j}
\end{array}\right],
$$

where $\mathbf{h}_{N+1}:=\left[\begin{array}{lll}h_{N+1,0}^{\top} & \ldots & h_{N+1, M}^{\top}\end{array}\right]^{\top}$ and $\mathbf{v}_{M+1}:=\left[\begin{array}{lll}v_{0, M+1}^{\top} & \ldots & v_{N, M+1}^{\top}\end{array}\right]^{\top}$, and the symmetric and positive semidefinite matrices $H \in \mathbb{R}^{\left[n_{h}(M+1)\right] \times\left[n_{h}(M+1)\right]}$ and $V \in \mathbb{R}^{\left[n_{v}(N+1)\right] \times\left[n_{v}(N+1)\right]}$ penalise the north-east boundary values of the horizontal and vertical semi-states, respectively. It is standard for the so-called Popov matrix in (4) to be chosen such that

$$
\Pi=\left[\begin{array}{cc}
Q & S \\
S^{\top} & R
\end{array}\right]=\Pi^{\top} \geq 0
$$


where the matrices $Q \in \mathbb{R}^{\left(n_{h}+n_{v}\right) \times\left(n_{h}+n_{v}\right)}$ and $S \in \mathbb{R}^{\left(n_{h}+n_{v}\right) \times m}$ are structured conformably with the semi-state vectors $h$ and $v$; specifically,

$$
Q=\left[\begin{array}{ll}
Q_{1,1} & Q_{1,2} \\
Q_{1,2}^{\top} & Q_{2,2}
\end{array}\right] \text { and } S=\left[\begin{array}{c}
S_{1} \\
S_{2}
\end{array}\right]
$$

where $Q_{1,1} \in \mathbb{R}^{n_{h} \times n_{h}}, Q_{1,2} \in \mathbb{R}^{n_{v} \times n_{v}}, Q_{2,2} \in \mathbb{R}^{n_{v} \times n_{v}}, S_{1} \in \mathbb{R}^{n_{h} \times m}$ and $S_{2} \in$ $\mathbb{R}^{n_{v} \times m}$. Note that there is no need to assume at this stage that $R=R^{\top} \geq 0$ is non-singular.

Rather than simply penalising the north-east boundary values of the semistates, it can be useful to require that these terminal values satisfy particular constraints. To this end, let $p_{j} \in\left\{0, \ldots, n_{h}\right\}$ and $W_{j} \in \mathbb{R}^{p_{j} \times n_{h}}$ be full row-rank for $j \in[0, M]$. Also let $q_{i} \in\left\{0, \ldots, n_{v}\right\}$ and $Z_{i} \in \mathbb{R}^{q_{i} \times n_{v}}$ be full row-rank for $i \in[0, N]$. Given $w_{j} \in \mathbb{R}^{p_{j}}$ and $z_{i} \in \mathbb{R}^{q_{i}}$, consider the additional constraints

$$
W_{j} h_{N+1, j}=w_{j} \quad \text { for } \quad j \in[0, M]
$$

on the horizontal semi-state and

$$
Z_{i} v_{i, M+1}=z_{i} \quad \text { for } \quad i \in[0, N]
$$

on the vertical semi-state. Note that if $p_{j}=0$ for a given $j \in[0, M]$, the matrix $W_{j}$ has zero rows, and the vector $w_{j}$ has zero entries. In this situation, the constraint (5) is vacuous; i.e., all the components of the vector $h_{N+1, j}$ are unconstrained. Likewise, if $q_{i}=0$ for $i \in[0, N]$, then $Z_{i}$ has zero rows, and $z_{i}$ has zero entries, so that all components of $v_{i, M+1}$ are unconstrained. The formulation also covers the double-ended boundary value problem, whereby all components of the vectors $h_{N+1, j}$ and $v_{i, M+1}$ are fixed for all $j \in[0, M]$ and $i \in[0, N]$, by setting $p_{j}=n_{h}$ for all $j \in[0, M]$ and $q_{i}=n_{v}$ for all $i \in[0, N]$. In this case, the matrices $H$ and $V$ in the performance index (4) can be set to zero, since the quadratic penalty on each $h_{N+1, j}$ and $v_{i, M+1}$ leads to a constant offset in the cost. In any cases, the terminal semi-state subspaces defined by the boundary conditions (5-6) must be reachable over the finite frame of interest, otherwise the set of admissible controls is empty. See (Kaczorek, 1985) for discussion of such issues. With these notions established it is possible to formalise the problem considered in the first part of the paper.

Problem 2.1 Find a control input u, over the finite-frame $\mathcal{Q}$, to minimise the quadratic performance index (4), subject to (1) and the boundary conditions (3), (5) and (6). 


\section{Open-loop optimal control}

In this section, three important issues related to the open-loop solution of Problem 2.1 are addressed. First, we present a generalised version of the optimality conditions established in ( $\mathrm{Li}$ and Fadali, 1991) in order to accomodate the north-east affine constraints (5-6). It is shown that the optimality conditions are not only necessary, but also sufficient. Open-loop computation of the optimal control is then discussed in detail and a compact expression is derived for the optimal cost.

\subsection{Characterising Optimality}

In (Li and Fadali, 1991), necessary optimality conditions are obtained for Problem 2.1 in the absence of the north-east boundary constraints (5-6), as a special case of a result established therein for a non-linear version of the constraint (1). The conditions were obtained via a variational analysis and the question of sufficiency remained unanswered. A similar analysis yields the following necessary conditions for Problem 2.1 as stated in terms of (5-6).

Proposition 3.1 Given a control input $u$, defined over the finite frame $\mathcal{Q}$, suppose that (5-6) are satisfied by the corresponding semi-state signals $h$ and $v$ generated via (1) and (3), over the finite frames $\mathcal{H}$ and $\mathcal{V}$, respectively. If $u$ minimises (4) then there exist $\lambda_{i, j} \in \mathbb{R}^{n_{h}}$ for $(i, j) \in \mathcal{H}, \mu_{i, j} \in \mathbb{R}^{n_{v}}$ for $(i, j) \in \mathcal{V}$, $\xi_{j} \in \mathbb{R}^{p_{j}}$ for $j \in[0, M]$ and $\zeta_{i} \in \mathbb{R}^{q_{i}}$ for $i \in[0, N]$ such that:

(i) for all $(i, j) \in \mathcal{Q}$

$$
\begin{aligned}
& {\left[\begin{array}{l}
\lambda_{i, j} \\
\mu_{i, j}
\end{array}\right]=Q\left[\begin{array}{l}
h_{i, j} \\
v_{i, j}
\end{array}\right]+S u_{i, j}+A^{\top}\left[\begin{array}{l}
\lambda_{i+1, j} \\
\mu_{i, j+1}
\end{array}\right],} \\
& 0=R u_{i, j}+S^{\top}\left[\begin{array}{l}
h_{i, j} \\
v_{i, j}
\end{array}\right]+B^{\top}\left[\begin{array}{l}
\lambda_{i+1, j} \\
\mu_{i, j+1}
\end{array}\right], \\
& {\left[\begin{array}{l}
h_{i+1, j} \\
v_{i, j+1}
\end{array}\right]=A\left[\begin{array}{l}
h_{i, j} \\
v_{i, j}
\end{array}\right]+B u_{i, j} ;}
\end{aligned}
$$

(ii) for all $j \in[0, M]$

$$
\begin{aligned}
h_{0, j} & =a_{j} \quad \text { and } \quad W_{j} h_{N+1, j}=w_{j}, \\
\lambda_{N+1, j} & =\sum_{k=0}^{M} H_{j, k} h_{N+1, j}+W_{j}^{\top} \xi_{j},
\end{aligned}
$$


where $H_{i, j}$ denotes the $(i, j)$-th $n_{h} \times n_{h}$ sub-block matrix of the penalty matrix $H$ for $i, j \in[0, M]$;

(iii) for all $i \in[0, N]$

$$
\begin{aligned}
v_{i, 0} & =b_{i} \quad \text { and } \quad Z_{i} v_{i, M+1}=z_{i}, \\
\mu_{i, M+1} & =\sum_{l=0}^{N} V_{l, i} v_{i, M+1}+Z_{i}^{\top} \zeta_{i} ;
\end{aligned}
$$

where $V_{i, j}$ denotes the $(i, j)$-th $n_{v} \times n_{v}$ sub-block matrix of the penalty matrix $V$ for $i, j \in[0, M]$.

Proof: The extension of the proof in (Li and Fadali, 1991) to accommodate the constraints (5-6) is straightforward.

It is now established that the conditions in Proposition 3.1 are not only necessary, but also sufficient for optimality. The approach taken is similar in nature to that of (Mangasarian, 1966), in that convexity is exploited.

Proposition 3.2 If the boundary value problem (7-13) admits a solution, the corresponding control input $u$ over the finite frame $\mathcal{Q}$ solves Problem 2.1 in the sense that it minimises (4) subject to the constraints (1), (3) and (5-6).

Proof: Let $h_{i, j}, v_{i, j}, u_{i, j}, \lambda_{i, j}, \mu_{i, j}, \xi_{j}$ and $\zeta_{i}$ be such that equations (11-12) hold and let $\hat{h}_{i, j}, \hat{v}_{i, j}, \hat{u}_{i, j}$ be such that all of the constraints of the optimal control problem are satisfied; i.e.

$$
\begin{aligned}
{\left[\begin{array}{c}
\hat{h}_{i+1, j} \\
\hat{v}_{i, j+1}
\end{array}\right] } & =A\left[\begin{array}{c}
\hat{h}_{i, j} \\
\hat{v}_{i, j}
\end{array}\right]+B \hat{u}_{i, j} \quad \forall(i, j) \in \mathcal{Q}, \\
\hat{h}_{0, j} & =a_{j} \quad \text { and } W_{j} \hat{h}_{N+1, j}=w_{j}, \quad j \in[0, M], \\
\hat{v}_{i, 0} & =b_{i} \quad \text { and } Z_{i} \hat{v}_{i, M+1}=z_{i}, \quad i \in[0, N] .
\end{aligned}
$$

Let $J$ denote the value of the quadratic costs (4) for the input $u$ (and corresponding $h$ and $v$ ) and let $\hat{J}$ denote the value for the input $\hat{u}$ (and correspond$\operatorname{ing} \hat{h}$ and $\hat{v}$ ). In what follows, it is shown that $J \leq \hat{J}$. By defining the vectors $p_{i, j}:=\left[\begin{array}{lll}h_{i, j}^{\top} & v_{i, j}^{\top} & u_{i, j}^{\top}\end{array}\right]^{\top}, \hat{p}_{i, j}:=\left[\hat{h}_{i, j}^{\top} \hat{v}_{i, j}^{\top} \hat{u}_{i, j}^{\top}\right]^{\top}, \hat{\mathbf{h}}_{N+1}:=\left[\hat{h}_{N+1,0}^{\top} \ldots \hat{h}_{N+1, M}^{\top}\right]^{\top}$ and $\hat{\mathbf{v}}_{M+1}:=\left[\hat{v}_{0, M+1}^{\top} \cdots \hat{v}_{N, M+1}^{\top}\right]^{\top}$, one obtains

$$
\hat{J}-J=\hat{\mathbf{h}}_{N+1}^{\top} H \hat{\mathbf{h}}_{N+1}+\hat{\mathbf{v}}_{M+1}^{\top} V \hat{\mathbf{v}}_{M+1} \mathbf{v}_{M+1}^{\top} V \mathbf{v}_{M+1}+\sum_{(i, j) \in \mathcal{Q}}\left(\hat{p}_{i, j}^{\top} \Pi \hat{p}_{i, j}-p_{i, j}^{\top} \Pi p_{i, j}\right) .
$$


Now, by the positive semi-definiteness of the quadratic forms $\left(\mathbf{h}_{N+1}-\hat{\mathbf{h}}_{N+1}\right)^{\top} H\left(\mathbf{h}_{N+1}-\right.$ $\left.\hat{\mathbf{h}}_{N+1}\right)$ and $\left(\mathbf{v}_{M+1}-\hat{\mathbf{v}}_{M+1}\right)^{\top} V\left(\mathbf{v}_{M+1}-\hat{\mathbf{v}}_{M+1}\right)$,

$$
\begin{aligned}
& \hat{\mathbf{h}}_{N+1}^{\top} H \hat{\mathbf{h}}_{N+1}-\mathbf{h}_{N+1}^{\top} H \mathbf{h}_{N+1} \geq 2 \mathbf{h}_{N+1}^{\top} H\left(\hat{\mathbf{h}}_{N+1}-\mathbf{h}_{N+1}\right) \text { and } \\
& \hat{\mathbf{v}}_{M+1}^{\top} V \hat{\mathbf{v}}_{M+1}-\mathbf{v}_{M+1}^{\top} V \mathbf{v}_{M+1} \geq 2 \mathbf{v}_{M+1}^{\top} V\left(\hat{\mathbf{v}}_{M+1}-\mathbf{v}_{M+1}\right) .
\end{aligned}
$$

Likewise $\hat{p}_{i, j}^{\top} \Pi \hat{p}_{i, j}-p_{i, j}^{\top} \Pi p_{i, j} \geq 2 p_{i, j}^{\top} \Pi\left(\hat{p}_{i, j}-p_{i, j}\right)$ for all $(i, j) \in \mathcal{Q}$, so that

$$
\hat{J}-J \geq 2 \mathbf{h}_{N+1}^{\top} H\left(\hat{\mathbf{h}}_{N+1}-\mathbf{h}_{N+1}\right)+2 \mathbf{v}_{M+1}^{\top} V\left(\hat{\mathbf{v}}_{M+1}-\mathbf{v}_{M+1}\right)+\sum_{(i, j) \in \mathcal{Q}} 2 p_{i, j}^{\top} \Pi\left(\hat{p}_{i, j}-p_{i, j}\right) .
$$

By virtue of $(7)$ it follows that for any $(i, j) \in \mathcal{Q}$,

$$
\begin{aligned}
& p_{i, j}^{\top} \Pi= \\
& {\left[\begin{array}{ll}
\left.\lambda_{i, j}^{\top}-\lambda_{i+1, j}^{\top} A_{1,1}-\mu_{i, j+1}^{\top} A_{2,1} \quad \mu_{i, j}^{\top}-\lambda_{i+1, j}^{\top} A_{1,2}-\mu_{i, j+1}^{\top} A_{2,2} \quad-\lambda_{i+1, j}^{\top} B_{1}-\mu_{i, j+1}^{\top} B_{2}\right] .
\end{array}\right.}
\end{aligned}
$$

As such,

$$
\begin{aligned}
p_{i, j}^{\top} \Pi\left(\hat{p}_{i, j}-p_{i, j}\right)= & \lambda_{i+1, j}^{\top} h_{i+1, j}-\lambda_{i+1, j}^{\top} \hat{h}_{i+1, j}+\mu_{i, j+1}^{\top} v_{i, j+1}-\mu_{i, j+1}^{\top} \hat{v}_{i, j+1} \\
& +\lambda_{i, j}^{\top}\left(\hat{h}_{i, j}-h_{i, j}\right)+\mu_{i, j}^{\top}\left(\hat{v}_{i, j}-v_{i, j}\right),
\end{aligned}
$$

where equation (9) has been used. By (11) it then follows that

$$
\begin{aligned}
\sum_{(i, j) \in \mathcal{Q}} \lambda_{i+1, j}^{\top} & \left(h_{i+1, j}-\hat{h}_{i+1, j}\right)-\lambda_{i, j}^{\top}\left(h_{i, j}-\hat{h}_{i, j}\right) \\
& =\sum_{j=0}^{M} \lambda_{N+1, j}^{\top}\left(h_{N+1, j}-\hat{h}_{N+1, j}\right)-\sum_{j=0}^{M} \lambda_{0, j}^{\top}\left(h_{0, j}-\hat{h}_{0, j}\right) \\
& =\sum_{j=0}^{M}\left(\sum_{k=0}^{M} h_{N+1, j}^{\top} H_{j, k}^{\top}+\xi_{j}^{\top} W_{j}\right)\left(h_{N+1, j}-\hat{h}_{N+1, j}\right)-\sum_{j=0}^{M} \lambda_{0, j}^{\top}\left(h_{0, j}-\hat{h}_{0, j}\right) \\
& =-\mathbf{h}_{N+1}^{\top} H\left(\hat{\mathbf{h}}_{N+1}-\mathbf{h}_{N+1}\right)-\sum_{j=0}^{M} \lambda_{0, j}^{\top}\left(h_{0, j}-\hat{h}_{0, j}\right),
\end{aligned}
$$

since both $\xi_{j}^{\top} W_{j} h_{N+1, j}$ and $\xi_{j}^{\top} W_{j} \hat{h}_{N+1, j}$ equal $w_{j}$ for all $j \in[0, M]$. In a similar way, it is can be shown that $\sum_{(i, j) \in \mathcal{Q}} \mu_{i, j+1}^{\top}\left(v_{i, j+1}-\hat{v}_{i, j+1}\right)-\mu_{i, j}^{\top}\left(v_{i, j}-\right.$ $\left.\hat{v}_{i, j}\right)=-\mathbf{v}_{M+1}^{\top} V\left(\hat{\mathbf{v}}_{M+1}-\mathbf{v}_{M+1}\right)-\sum_{i=0}^{N} \mu_{i, 0}^{\top}\left(v_{i, 0}-\hat{v}_{i, 0}\right)$, so that

$$
\hat{J}-J \geq-\sum_{j=0}^{M} \lambda_{0, j}^{\top}\left(h_{0, j}-\hat{h}_{0, j}\right)-\sum_{i=0}^{N} \mu_{i, 0}^{\top}\left(v_{i, 0}-\hat{v}_{i, 0}\right),
$$

the right hand-side of which is zero in view of (10-12) and (15). 
Remark 3.1 As in the 1-D case, any constraint on components of the semistate vector correspond to components of the semi-costate vector that are unconstrained (and vice-versa). In fact, due to the constraint $W_{j} h_{N+1, j}=w_{j}$, the components of the corresponding semi-costate vector $\lambda_{N+1, j}$ are constrained on the subspace ker $W_{j}$. The freedom of the remaining components is represented by the components of the vector $\xi_{j}$. In particular:

- when all the components of the semi-state $h_{N+1, j}$ are unconstrained, i.e., when $p_{j}=0$, we can assume ker $W_{j}=\mathbb{R}^{n_{h}}$, which implies that all the components of the semi-costate $\lambda_{N+1, j}$ are constrained, and $\xi_{j}$ disappears (or, more precisely, it is a vector of $p_{j}=0$ entries);

- when all the components of the semi-state $h_{N+1, j}$ are constrained, i.e., when $p_{j}=n_{h}$, then $\operatorname{ker} W_{j}=\{0\}$ since $W_{j}$ is invertible, and $\xi_{j}$ is an $n_{h}$-dimensional vector. It follows that there are no constraints on $\lambda_{N+1, j}$, since (11) gives rise to $n_{h}$ equations, but the number of free variables equals the number of components of $\xi_{j}$, i.e., $n_{h}$.

Analogous remarks apply to the constraints on the vertical semi-state.

Remark 3.2 Let us consider the case where $p_{j}=0$ for all $j \in[0, M]$ and $q_{i}=0$ for all $i \in[0, N]$, i.e., where there are no constraints on the semi-states $h_{N+1, j}$ and $v_{i, M+1}$ for $j \in[0, M]$ and $i \in[0, N]$. Since in this case any control input to (1) is admissible for the specified boundary conditions (3), in the sense that a corresponding semi-state trajectory always exists, Problem 2.1 can be re-expressed as a feasible static quadratic optimisation problem in the $l=m[(N+1) \cdot(M+1)]$ unconstrained variables representing the control input on $\mathcal{Q}$. In particular, Problem 2.1 always admits solutions in this case. Thus, by virtue of Theorem 3.2, the boundary value problem in the semi-state and semi-costate vectors (7-13) always admits solutions. In the next section, the computation of such solutions is discussed in more detail. When the constraints (5-6) are imposed, on the other hand, Problem 2.1 admits solutions if and only if there exists a trajectory of (1) that satisfies (3) and (5-6).

\subsection{Computing optimal open-loop controls}

In general, the boundary value problem (7-13) is difficult to solve. In this section it is shown that, under some assumptions on the problem matrices specifically $R>0$ and $A-B R^{-1} S^{\top}$ non-singular - equations (7), (8) and (9) can be re-expressed in the form of a standard Roesser model. The set of solutions can then be written in a parameterised form, using the transition matrix recursion given in (Roesser, 1975). It is shown how to impose the boundary conditions (11) and (13) to determine the parameters involved. This leads to 
a simple linear equation for the parameters, which must admit solutions in order for the optimal control problem to be feasible. When a solution for the parameters exists, the corresponding input and semi-state functions are optimal by construction, as they satisfy the sufficient (and necessary) conditions in Proposition 3.2.

When $R$ is positive definite (and hence invertible), equation (8) can be solved for $u_{i, j}$, so that (7) and (9) can be written together as

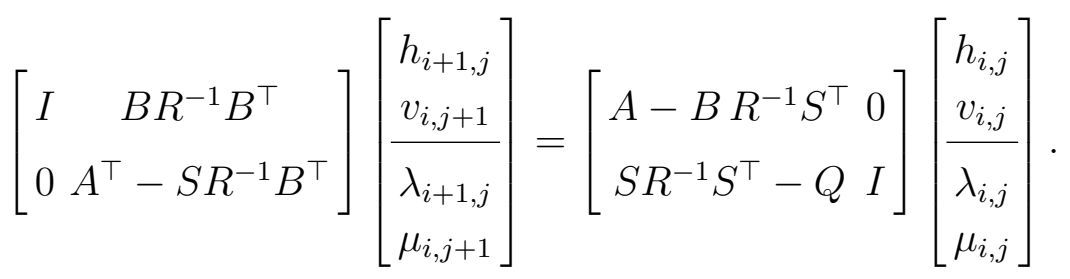

The matrices appearing above are invertible iff $A-B R^{-1} S^{\top}$ is invertible. In this case, (17) can be rewritten as

$$
\left[\begin{array}{c}
r_{i+1, j} \\
s_{i, j+1}
\end{array}\right]=\left[\begin{array}{cc}
P_{1} & P_{2} \\
P_{3} & P_{4}
\end{array}\right]\left[\begin{array}{l}
r_{i, j} \\
s_{i, j}
\end{array}\right],
$$

where $r_{i, j}:=\left[\begin{array}{c}h_{i, j} \\ \lambda_{i, j}\end{array}\right], s_{i, j}:=\left[\begin{array}{c}v_{i, j} \\ \mu_{i, j}\end{array}\right]$ and $\left[\begin{array}{ll}P_{1} & P_{2} \\ P_{3} & P_{4}\end{array}\right]:=\left[\begin{array}{ll}G_{1,1}^{\prime} & G_{1,2}^{\prime \prime} \\ G_{2,1}^{\prime \prime} & G_{2,2}^{\prime}\end{array}\right]^{-1}\left[\begin{array}{ll}T_{1,1}^{\prime} & T_{1,2}^{\prime \prime} \\ T_{2,1}^{\prime \prime} & T_{2,2}^{\prime}\end{array}\right]$, with

$$
\begin{array}{rlr}
G_{k, l}^{\prime}:=\left[\begin{array}{cc}
I & B_{k} R^{-1} B_{l}^{\top} \\
0 & A_{k, l}^{\top}-S_{k} R^{-1} B_{l}^{\top}
\end{array}\right], & G_{k, l}^{\prime \prime}:=\left[\begin{array}{cc}
0 & B_{k} R^{-1} B_{l}^{\top} \\
0 & A_{k, l}^{\top}-S_{k} R^{-1} B_{l}^{\top}
\end{array}\right], \\
T_{k, l}^{\prime}:=\left[\begin{array}{cc}
A_{k, l}-B_{k} R^{-1} S_{l}^{\top} & 0 \\
S_{k} R^{-1} S_{l}^{\top}-Q_{k, l} & I
\end{array}\right], & T_{k, l}^{\prime \prime}:=\left[\begin{array}{cc}
A_{k, l}-B_{k} R^{-1} S_{l}^{\top} & 0 \\
S_{k} R^{-1} S_{l}^{\top}-Q_{k, l} & 0
\end{array}\right] .
\end{array}
$$

Model (18) is in Roesser form. As such, the semi-state transition matrix associated with (18) can be computed by using the general response formula (Roesser, 1975):

$$
\begin{aligned}
& \Phi_{0,0}=I \\
& \Phi_{i, j}=0 \quad \text { if } i<0 \text { or } j<0 ; \\
& \Phi_{i, j}=\Phi_{1,0} \Phi_{i-1, j}+\Phi_{0,1} \Phi_{i, j-1} \quad \forall(i, j) \in \mathbb{N} \backslash\{(0,0)\}
\end{aligned}
$$

where $\Phi_{1,0}:=\left[\begin{array}{cc}P_{1} & P_{2} \\ 0 & 0\end{array}\right]$ and $\Phi_{0,1}:=\left[\begin{array}{cc}0 & 0 \\ P_{3} & P_{4}\end{array}\right]$. Now, partitioning $\Phi_{i, j}$ as $\Phi_{i, j}=$ $\left[\begin{array}{cc}\Phi_{i, j}^{1} & \Phi_{i, j}^{2} \\ \Phi_{i, j}^{3} & \Phi_{i, j}^{4}\end{array}\right]$, conformably with $\left[\begin{array}{l}r_{i, j} \\ s_{i, j}\end{array}\right]$, where each $\Phi_{i, j}^{k}$, for $k \in\{1,2,3,4\}$, is in 
turn partitioned as $\Phi_{i, j}^{k}=\left[\begin{array}{l}\Phi_{i, j}^{k, 1} \Phi_{i, j}^{k, 2} \\ \Phi_{i, j}^{k, 3} \Phi_{i, j}^{k, 4}\end{array}\right]$, conformably with $\left[\begin{array}{c}h_{i, j} \\ \lambda_{i, j}\end{array}\right]$ and $\left[\begin{array}{c}v_{i, j} \\ \mu_{i, j}\end{array}\right]$, we can write the solution of (18) explicitly as

$$
\begin{aligned}
h_{i, j} & =\sum_{k=0}^{j}\left(\Phi_{i, j-k}^{1,1} a_{k}+\Phi_{i, j-k}^{1,2} \lambda_{0, k}\right)+\sum_{h=0}^{i}\left(\Phi_{i-h, j}^{2,1} b_{h}+\Phi_{i-h, j}^{2,2} \mu_{h, 0}\right), \\
\lambda_{i, j} & =\sum_{k=0}^{j}\left(\Phi_{i, j-k}^{1,3} a_{k}+\Phi_{i, j-k}^{1,4} \lambda_{0, k}\right)+\sum_{h=0}^{i}\left(\Phi_{i-h, j}^{2,3} b_{h}+\Phi_{i-h, j}^{2,4} \mu_{h, 0}\right), \\
v_{i, j} & =\sum_{k=0}^{j}\left(\Phi_{i, j-k}^{3,1} a_{k}+\Phi_{i, j-k}^{3,2} \lambda_{0, k}\right)+\sum_{h=0}^{i}\left(\Phi_{i-h, j}^{4,1} b_{h}+\Phi_{i-h, j}^{4,2} \mu_{h, 0}\right), \\
\mu_{i, j}= & \sum_{k=0}^{j}\left(\Phi_{i, j-k}^{3,3} a_{k}+\Phi_{i, j-k}^{3,4} \lambda_{0, k}\right)+\sum_{h=0}^{i}\left(\Phi_{i-h, j}^{4,3} b_{h}+\Phi_{i-h, j}^{4,4} \mu_{h, 0}\right),
\end{aligned}
$$

where the boundary conditions (3) have been used. From these semi-state trajectories, which satisfy (3), we need to select those that also satisfy the remaining equations in Theorem 3.2.

Suppose for the moment that the penalty matrices $H$ and $V$ are diagonal; i.e., $H=\operatorname{diag}\left\{H_{0}, H_{1}, \ldots, H_{M}\right\}$, where $H_{j} \in \mathbb{R}^{n_{h}}$ for all $j \in\{0, \ldots, M\}$, and $V=\operatorname{diag}\left\{V_{0}, V_{1}, \ldots, V_{N}\right\}$, where $V_{i} \in \mathbb{R}^{n_{v}}$ for all $i \in\{0, \ldots, N\}$. Then, imposing (11) on (19) and (20) gives

$$
\begin{aligned}
\lambda_{N+1, j}= & \sum_{k=0}^{j}\left(\Phi_{N+1, j-k}^{1,3} a_{k}+\Phi_{N+1, j-k}^{1,4} \lambda_{0, k}\right)+\sum_{h=0}^{N}\left(\Phi_{N+1-h, j}^{2,3} b_{h}+\Phi_{N+1-h, j}^{2,4} \mu_{h, 0}\right) \\
= & H_{j}\left(\sum_{k=0}^{j}\left(\Phi_{N+1, j-k}^{1,1} a_{k}+\Phi_{N+1, j-k}^{1,2} \lambda_{0, k}\right)+\sum_{h=0}^{N}\left(\Phi_{N+1-h, j}^{2,1} b_{h}+\Phi_{N+1-h, j}^{2,2} \mu_{h, 0}\right)\right) \\
& +W_{j}^{\top} \xi_{j} .
\end{aligned}
$$

Let $j \in[0, M]$ be such that the full row-rank matrix $W_{j}$ is not square, i.e., ker $W_{j} \neq\{0\}$, and let $\tilde{W}_{j}$ be a basis matrix for the null-space of $W_{j}$, so that $\tilde{W}_{j}^{\top} W_{j}^{\top}=0$. The by premultiplying (23) by $\tilde{W}_{j}^{\top}$ we obtain

$$
\begin{aligned}
& \tilde{W}_{j}^{\top}\left[\sum_{k=0}^{j}\left(\Phi_{N+1, j-k}^{1,4}-H_{j, j} \Phi_{N+1, j-k}^{1,2}\right) \lambda_{0, k}+\sum_{h=0}^{N}\left(\Phi_{N+1-h, j}^{2,4}-H_{j, j} \Phi_{N+1-h, j}^{2,2}\right) \mu_{h, 0}\right]= \\
& -\tilde{W}_{j}^{\top}\left[\sum_{k=0}^{j}\left(\Phi_{N+1, j-k}^{1,3}-H_{j, j} \Phi_{N+1, j-k}^{1,1}\right) a_{k}+\sum_{h=0}^{N}\left(\Phi_{N+1-h, j}^{2,3}-H_{j, j} \Phi_{N+1-h, j}^{2,1}\right) b_{h}\right] .
\end{aligned}
$$

Equation (24) represents $n_{h}-p_{j}$ equations in the $\left[(M+1) n_{h}\right] \times\left[(N+1) n_{v}\right]$ unknowns $\lambda_{0,0}, \ldots, \lambda_{0, M}, \mu_{0,0}, \ldots, \mu_{N, 0}$. Imposing $W_{j} h_{N+1, j}=w_{j}$ on (19) gives 


$$
W_{j}\left[\sum_{k=0}^{j}\left(\Phi_{N+1, j-k}^{2,1} a_{k}+\Phi_{N+1, j-k}^{1,2} \lambda_{0, k}\right)+\sum_{h=0}^{N+1}\left(\Phi_{N+1-h, j}^{2,1} b_{h}+\Phi_{N+1-h, j}^{2,2} \mu_{h, 0}\right)\right]=w_{j}
$$

which represents $p_{j}$ equations in the $\left[(M+1) n_{h}\right] \times\left[(N+1) n_{v}\right]$ unknowns $\lambda_{0,0}, \ldots, \lambda_{0, M}, \mu_{0,0}, \ldots, \mu_{N, 0}$. Similarly, (13) and (21-22) yield

$$
\begin{aligned}
& \mu_{i, M+1}=\sum_{k=0}^{M}\left(\Phi_{i, M+1-k}^{3,3} a_{k}+\Phi_{i, M+1-k}^{3,4} \lambda_{0, k}\right)+\sum_{h=0}^{i}\left(\Phi_{i-h, M+1}^{4,3} b_{h}+\Phi_{i-h, M+1}^{4,4} \mu_{h, 0}\right) \\
& =V_{i}\left(\sum_{k=0}^{M}\left(\Phi_{i, M+1-k}^{3,1} a_{k}+\Phi_{i, M+1-k}^{3,2} \lambda_{0, k}\right)+\sum_{h=0}^{i}\left(\Phi_{i-h, M+1}^{4,1} b_{h}+\Phi_{i-h, M+1}^{4,2} \mu_{h, 0}\right)\right)+Z_{i}^{\top} \zeta_{i} .
\end{aligned}
$$

Let $i \in[0, N]$ be such that $Z_{i}$ is not square, i.e., $\operatorname{ker} Z_{i} \neq\{0\}$, and let $\tilde{Z}_{i}$ be, for all $i \in[0, N]$, a basis matrix for the null-space of $Z_{i}$, so that $\tilde{Z}_{i}^{\top} Z_{i}^{\top}=0$. Now by premultiplying by $\tilde{Z}_{i}^{\top}$, we get

$$
\begin{aligned}
& \tilde{Z}_{i}^{\top}\left[\sum_{k=0}^{M}\left(\Phi_{i, M+1-k}^{3,4}-V_{i} \Phi_{i, M+1-k}^{3,2}\right) \lambda_{0, k}+\sum_{h=0}^{i}\left(\Phi_{i, M+1-k}^{4,4}-V_{i} \Phi_{i, M+1-k}^{4,2}\right) \mu_{h, 0}\right]= \\
& -\tilde{Z}_{i}^{\top}\left[\sum_{k=0}^{M}\left(\Phi_{i, M+1-k}^{3,3}-V_{i} \Phi_{i, M+1-k}^{3,1}\right) a_{k}+\sum_{h=0}^{i}\left(\Phi_{i, M+1-k}^{4,3}-V_{i} \Phi_{i, M+1-k}^{4,1}\right) b_{h}\right] .
\end{aligned}
$$

Imposing $Z_{i} v_{i, M+1}=z_{i}$ on (21) gives

$Z_{i}\left[\sum_{k=0}^{M}\left(\Phi_{i, M+1-k}^{3,2} \lambda_{0, k}+\Phi_{i, M+1-k}^{3,1} a_{k}\right)+\sum_{h=0}^{i}\left(\Phi_{i-h, M+1}^{4,2} \mu_{h, 0}+\Phi_{i-h, M+1}^{4,1} b_{h}\right)\right]=z_{i}$

Equations (24), (25), (26) and (27) can be expressed in the following compact linear form

$$
\left[\begin{array}{c|c}
\Psi_{\lambda} & \Psi_{\mu} \\
\hline \Xi_{\lambda} & \Xi_{\mu}
\end{array}\right]\left[\begin{array}{c}
\lambda_{0,0} \\
\vdots \\
\frac{\lambda_{0, M}}{\mu_{0,0}} \\
\vdots \\
\mu_{N, 0}
\end{array}\right]=-\left[\begin{array}{l|l}
\Theta_{a} & \Theta_{b} \\
\hline \Gamma_{a} & \Gamma_{b}
\end{array}\right]\left[\begin{array}{c}
a_{0} \\
\vdots \\
\frac{a_{M}}{b_{0}} \\
\vdots \\
b_{N}
\end{array}\right]+\left[\begin{array}{c}
\hat{w}_{0} \\
\vdots \\
\hat{w}_{M} \\
\hat{z}_{0} \\
\vdots \\
\hat{z}_{N}
\end{array}\right]
$$

where

$$
\hat{w}_{j}:=\left[\begin{array}{c}
0_{n_{h}-p_{j}} \\
w_{j}
\end{array}\right] \text { for } j \in[0, M], \quad \hat{z}_{i}:=\left[\begin{array}{c}
0_{n_{v}-q_{i}} \\
z_{i}
\end{array}\right] \text { for } i \in[0, N],
$$


the matrices $\Omega_{i, j}$, for $i, j \in\{1,2\}$, are given by

$$
\begin{aligned}
& \Psi_{\lambda}:=\left[\begin{array}{cccc}
\Psi_{N+1,0,0}^{1} & 0 & \ldots & 0 \\
\Psi_{N+1,1,1}^{1} & \Psi_{N+1,0,1}^{1} & \ldots & 0 \\
\vdots & \vdots & \ddots & \vdots \\
\Psi_{N+1, M, M}^{1} \Psi_{N+1, M-1, M}^{1} & \ldots & \Psi_{N+1,0, M}^{1}
\end{array}\right], \quad \Psi_{\mu}:=\left[\begin{array}{cccc}
\Psi_{N+1,0,0}^{2} & \Psi_{N, 0,0}^{2} & \ldots & \Psi_{1,0,0}^{2} \\
\Psi_{N+1,1,1}^{2} & \Psi_{N, 1,1}^{2} & \ldots & \Psi_{1,1,1}^{2} \\
\vdots & \vdots & \ddots & \vdots \\
\Psi_{N+1, M, M}^{2} & \Psi_{N, M, M}^{2} & \ldots & \Psi_{1, M, M}^{2}
\end{array}\right], \\
& \Xi_{\lambda}:=\left[\begin{array}{cccc}
\Xi_{0, M+1,0}^{3} & \Xi_{0, M, 0}^{3} & \ldots & \Xi_{0,1,0}^{3} \\
\Xi_{1, M+1,1}^{3} & \Xi_{1, M, 1}^{3} & \ldots & \Xi_{1,1,1}^{3} \\
\vdots & \vdots & \ddots & \vdots \\
\Xi_{N, M+1, M}^{3} & \Xi_{N, M, N}^{3} & \ldots & \Xi_{N, 1, N}^{3}
\end{array}\right], \quad \Xi_{\mu}:=\left[\begin{array}{cccc}
\Xi_{0, M+1,0}^{4} & 0 & \ldots & 0 \\
\Xi_{1, M+1,1}^{4} & \Xi_{0, M+1,1}^{4} & \ldots & 0 \\
\vdots & \vdots & \ddots & \vdots \\
\Xi_{N, M+1, N}^{4} \Xi_{N-1, M+1, N}^{4} & \ldots & \Xi_{0, M+1, N}^{4}
\end{array}\right],
\end{aligned}
$$

and the matrices $\Upsilon_{i, j}$, for $i, j \in\{1,2\}$, are given by

$$
\Theta_{a}:=\left[\begin{array}{cccc}
\Theta_{N+1,0,0}^{1} & 0 & \ldots & 0 \\
\Theta_{N+1,0,1}^{1} & \Theta_{N+1,1,1}^{1} & \ldots & 0 \\
\vdots & \vdots & \ddots & \vdots \\
\Theta_{N+1, M, M}^{1} \Theta_{N+1, M-1, M}^{1} & \ldots & \Theta_{N+1,0, M}^{1}
\end{array}\right], \quad \Theta_{b}:=\left[\begin{array}{cccc}
\Theta_{N+1,0,0}^{2} & \Theta_{N, 0,0}^{2} & \ldots & \Theta_{1,0,0}^{2} \\
\Theta_{N+1,1,1}^{2} & \Theta_{N, 1,1}^{2} & \ldots & \Theta_{1,1,1}^{2} \\
\vdots & \vdots & \ddots & \vdots \\
\Theta_{N+1, M, M}^{2} & \Theta_{N, M, M}^{2} & \ldots & \Theta_{1, M, M}^{2}
\end{array}\right],
$$$$
\Gamma_{a}:=\left[\begin{array}{cccc}
\Gamma_{0, M+1,0}^{3} & \Gamma_{0, M, 0}^{3} & \ldots & \Gamma_{0,1,0}^{3} \\
\Gamma_{1, M+1,1}^{3} & \Gamma_{1, M, 1}^{3} & \ldots & \Gamma_{1,1,1}^{3} \\
\vdots & \vdots & \ddots & \vdots \\
\Gamma_{N, M+1, N}^{3} & \Gamma_{N, M, N}^{3} & \ldots & \Gamma_{N, 1, N}^{3}
\end{array}\right], \quad \Gamma_{b}:=\left[\begin{array}{cccc}
\Gamma_{0, M+1,0}^{4} & 0 & \ldots & 0 \\
\Gamma_{1, M+1,1}^{4} & \Gamma_{0, M+1,1}^{4} & \ldots & 0 \\
\vdots & \vdots & \ddots & \vdots \\
\Gamma_{N, M+1, N}^{4} \Gamma_{N-1, M+1, N}^{4} & \ldots \Gamma_{0, M+1, N}^{4}
\end{array}\right],
$$

with

$$
\begin{array}{ll}
\Psi_{h, l, k}^{X}:=\left[\begin{array}{c}
\tilde{W}_{k}^{\top}\left(\Phi_{h, l}^{X, 4}-H_{k, k} \Phi_{h, l}^{X, 2}\right) \\
W_{k} \Phi_{h, l}^{X, 2}
\end{array}\right], & \Theta_{h, l, k}^{X}:=\left[\begin{array}{c}
\tilde{W}_{k}^{\top}\left(\Phi_{h, l}^{X, 3}-H_{k, k} \Phi_{h, l}^{X, 1}\right) \\
W_{k} \Phi_{h, l}^{X, 1}
\end{array}\right], \\
\Xi_{h, l, k}^{X}:=\left[\begin{array}{c}
\tilde{Z}_{k}^{\top}\left(\Phi_{h, l}^{X, 4}-V_{k, k} \Phi_{h, l}^{X, 2}\right) \\
Z_{k} \Phi_{h, l}^{X, 2}
\end{array}\right], & \Gamma_{h, l, k}^{X}:=\left[\begin{array}{c}
\tilde{Z}_{k}^{\top}\left(\Phi_{h, l}^{X, 3}-V_{k, k} \Phi_{h, l}^{X, 1}\right) \\
Z_{k} \Phi_{h, l}^{X, 1}
\end{array}\right],
\end{array}
$$

for $X \in\{1,2,3,4\}$. All matrices $\Psi_{i, j}^{h, l, k}$ and $\Theta_{i, j}^{h, l, k}$ have $n_{h}$ rows, while all matrices $\Xi_{i, j}^{h, l, k}$ and $\Gamma_{i, j}^{h, l, k}$ have $n_{v}$ rows. In fact, $W_{j}$ has $p_{j}$ rows and matrix $\tilde{W}_{j}^{\top}$ has $n_{h}-p_{j}$ rows, while $Z_{i}$ has $q_{i}$ rows and matrix $\tilde{Z}_{i}^{\top}$ has $n_{v}-q_{i}$ rows. It follows that $\Omega_{1,1}$ and $\Upsilon_{1,1}$ are $\left[(M+1) n_{h}\right] \times\left[(M+1) n_{h}\right], \Omega_{1,2}$ and $\Upsilon_{1,2}$ are $\left[(M+1) n_{h}\right] \times\left[(N+1) n_{h}\right], \Omega_{2,1}$ and $\Upsilon_{2,1}$ are $\left[(N+1) n_{v}\right] \times\left[(M+1) n_{v}\right]$, 
$\Omega_{2,2}$ and $\Upsilon_{2,2}$ are $\left[(N+1) n_{v}\right] \times\left[(N+1) n_{v}\right]$. As such, $(28)$ consists of $[(M+$ 1) $\left.n_{h}\right] \times\left[(N+1) n_{v}\right]$ equations in the $\left[(M+1) n_{h}\right] \times\left[(N+1) n_{v}\right]$ unknowns $\lambda_{0,0}, \ldots, \lambda_{0, M}, \mu_{0,0}, \ldots, \mu_{N, 0}$.

Given $\lambda_{0, k}$ and $\mu_{l, 0}$, for $k \in\{0, \ldots, M\}$ and $l \in\{0, \ldots, N\}$, that satisfy (28), the semi-state and corresponding semi-costate trajectories $h_{i, j}, v_{i, j}, \lambda_{i, j}$ and $\mu_{i, j}$ can be determined for $(i, j) \in \mathcal{Q}$ via (19-22). These trajectories satisfy the Roesser model (18), which is equivalent to (7-9) under the assumptions considered, and the boundary conditions (10-13). Finally, by using (8), a corresponding control input $u_{i, j}$ can be computed, for all $(i, j) \in \mathcal{Q}$, since $R$ is invertible by hypothesis here. The semi-state trajectories and the control obtained are optimal since, by construction, they satisfy all the sufficient (and necessary) conditions for optimality.

Remark 3.3 If $p_{j}=n_{h}$ for some $j \in[0, M]$, or $q_{i}=n_{v}$ for some $i \in[0, N]$, the corresponding equations (24) or (26) can be neglected. In this case, the first block-row of matrices $\Psi_{h, l, k}^{X}, \Theta_{h, l, k}^{X}, \Xi_{h, l, k}^{X}$ and $\Gamma_{h, l, k}^{X}$ disappear.

Remark 3.4 In view of Remark 3.2, when $p_{j}=0$ for some $j \in[0, M]$, we can take $\tilde{W}_{j}=I_{n_{h}}$. In this case, equation (25) disappears, and the vector $w_{j}$ has zero entries. Similarly, when $q_{i}=0$ for some $i \in[0, N]$, we can take $\tilde{Z}_{i}=I_{n_{v}}$, so that $(27)$ disappears, and $z_{j}$ has zero entries. In this case, the boundary value problem always admits solutions, and as such, given arbitrary choice of the boundary condition parameters $a_{i}$ and $b_{j}$, the linear equation (28) admits solutions; i.e., $\operatorname{im}\left[\begin{array}{ll}\Upsilon_{1,1} & \Upsilon_{1,2} \\ \Upsilon_{2,1} & \Upsilon_{2,2}\end{array}\right] \subseteq \operatorname{im}\left[\begin{array}{ll}\Omega_{1,1} & \Omega_{1,2} \\ \Omega_{2,1} & \Omega_{2,2}\end{array}\right]$, since $\hat{w}_{j}$ and $\hat{z}_{i}$ are all vectors with zero entries. Also note that in this case the solutions of (28) are parameterised in the kernel of $\left[\begin{array}{ll}\Omega_{1,1} & \Omega_{1,2} \\ \Omega_{2,1} & \Omega_{2,2}\end{array}\right]$.

Remark 3.5 When the matrices $H$ and $V$ are not diagonal, the procedure outlined above still applies with little modifications. In this case, we find that equations (24) and (26) become respectively

$$
\begin{aligned}
& \lambda_{N+1, j}=\sum_{k=0}^{j}\left(\Phi_{N+1, j-k}^{1,3} a_{k}+\Phi_{N+1, j-k}^{1,4} \lambda_{0, k}\right)+\sum_{h=0}^{N}\left(\Phi_{N+1-h, j}^{2,3} b_{h}+\Phi_{N+1-h, j}^{2,4} \mu_{h, 0}\right) \\
& =\sum_{k=0}^{M} H_{j, k}\left(\sum_{k=0}^{j}\left(\Phi_{N+1, j-k}^{1,1} a_{k}+\Phi_{N+1, j-k}^{1,2} \lambda_{0, k}\right)+\sum_{h=0}^{N}\left(\Phi_{N+1-h, j}^{2,1} b_{h}+\Phi_{N+1-h, j}^{2,2} \mu_{h, 0}\right)\right)+W_{j}^{\top} \xi_{j}
\end{aligned}
$$

and 


$$
\begin{aligned}
& \mu_{i, M+1}=\sum_{k=0}^{M}\left(\Phi_{i, M+1-k}^{3,3} a_{k}+\Phi_{i, M+1-k}^{3,4} \lambda_{0, k}\right)+\sum_{h=0}^{i}\left(\Phi_{i-h, M+1}^{4,3} b_{h}+\Phi_{i-h, M+1}^{4,4} \mu_{h, 0}\right) \\
& =\sum_{k=0}^{N} V_{k, i}\left(\sum_{k=0}^{M+1}\left(\Phi_{i, M+1-k}^{3,1} a_{k}+\Phi_{i, M+1-k}^{3,2} \lambda_{0, k}\right)+\sum_{h=0}^{i}\left(\Phi_{i-h, M+1}^{4,1} b_{h}+\Phi_{i-h, M+1}^{4,2} \mu_{h, 0}\right)\right)+Z_{i}^{\top} \zeta_{i} .
\end{aligned}
$$

These expressions still lead to a linear relation between $\lambda_{0,0}, \lambda_{0,0}, \lambda_{0,1}, \ldots, \lambda_{0, M}$, $\mu_{0,0}, \mu_{1,0}, \ldots, \mu_{N, 0}$ and $a_{0}, a_{1}, \ldots, a_{M}, b_{0}, b_{1}, \ldots, b_{N}$, so that equation (28) still holds, but the expression of the matrices $\Omega_{i, j}$ and $\Upsilon_{i, j}$ become more complex.

Remark 3.6 Clearly, the assumption that $A-B R^{-1} S^{\top}$ be invertible causes some loss of generality. On the other hand, both $R$ and $S$ are weighting matrices that are usually chosen by the designer to obtain a satisfactory behaviour of the semi-states and of the control input. Hence, in most cases - with possible problems arising when the pair $(A, B)$ has uncontrollable eigenvalues at zeromatrices $R$ and $S$ can be chosen so as to meet the condition on $A-B R^{-1} S^{\top}$.

Remark 3.7 When $Q=S R^{-1} S^{\top}$, or in the even more particular case of minimum energy control, as considered in (Li and Fadali, 1991, Section IV), where $Q$ and $S$ are both zero, equation (7) and the corresponding boundary condition can be solved 'backwards' as described in (Li and Fadali, 1991); this yields the solution presented in (Kaczorek and Klamka, 1986), which was established via quite different techniques.

\subsection{Optimal cost}

For the 1-D LQ optimal control problem, it is well-known that the optimal value of the performance index can be expressed in terms of the boundary values of the state and costate. This is particularly important when dealing with complex optimisation problems, for example more general (possibly parametric) optimisation problems having the LQ as a subproblem. For example, consider the case of two (or more) optimal control problems that are coupled by constraints or by a weight on the respective boundary conditions. With the following theorem it is established that a similar result holds for 2-D Roesser models.

Theorem 3.1 Let $h, v, u, \lambda$ and $\mu$ satisfy equations (7-13). The optimal cost $J^{\star}$ is given by

$$
\begin{gathered}
J^{\star}=\mathbf{h}_{N+1}^{\top} H \mathbf{h}_{N+1}+\mathbf{v}_{M+1}^{\top} V \mathbf{v}_{M+1}+\sum_{j=0}^{M}\left(h_{0, j}^{\top} \lambda_{0, j}-h_{N+1, j}^{\top} \lambda_{N+1, j}\right) \\
+\sum_{i=0}^{N}\left(v_{i, 0}^{\top} \mu_{i, 0}-v_{i, M+1}^{\top} \mu_{i, M+1}\right) .
\end{gathered}
$$


Proof: Let $x_{i, j}:=\left[\begin{array}{l}h_{i, j} \\ v_{i, j}\end{array}\right]$ and $c:=\sum_{(i, j) \in \mathcal{Q}}\left[\begin{array}{ll}x_{i, j}^{\top} & u_{i, j}^{\top}\end{array}\right] \Pi\left[\begin{array}{l}x_{i, j} \\ u_{i, j}\end{array}\right]$. By (8), (7) and (9) we get

$$
\begin{aligned}
c & =\sum_{(i, j) \in \mathcal{Q}} x_{i, j}^{\top} Q x_{i, j}+x_{i, j}^{\top} S u_{i, j}-u_{i, j}^{\top} B^{\top}\left[\begin{array}{l}
\lambda_{i+1, j} \\
\mu_{i, j+1}
\end{array}\right] \\
& =\sum_{(i, j) \in \mathcal{Q}} x_{i, j}^{\top}\left(\left[\begin{array}{c}
\lambda_{i, j} \\
\mu_{i, j}
\end{array}\right]-A^{\top}\left[\begin{array}{c}
\lambda_{i+1, j} \\
\mu_{i, j+1}
\end{array}\right]\right)-u_{i, j}^{\top} B^{\top}\left[\begin{array}{l}
\lambda_{i+1, j} \\
\mu_{i, j+1}
\end{array}\right] \\
& =\sum_{(i, j) \in \mathcal{Q}}\left[h_{i, j}^{\top} v_{i, j}^{\top}\right]\left[\begin{array}{l}
\lambda_{i, j} \\
\mu_{i, j}
\end{array}\right]-\left[h_{i+1, j}^{\top} v_{i, j+1}^{\top}\right]\left[\begin{array}{l}
\lambda_{i+1, j} \\
\mu_{i, j+1}
\end{array}\right]
\end{aligned}
$$

Since we have $\sum_{(i, j) \in \mathcal{Q}}\left(h_{i, j}^{\top} \lambda_{i, j}-h_{i+1, j}^{\top} \lambda_{i+1, j}\right)=\sum_{j=0}^{M} h_{0, j}^{\top} \lambda_{0, j}-h_{N+1, j}^{\top} \lambda_{N+1, j}$ and $\sum_{(i, j) \in \mathcal{Q}}\left(v_{i, j}^{\top} \mu_{i, j}-v_{i+1, j}^{\top} \mu_{i+1, j}\right)=\sum_{i=0}^{N} v_{i, 0}^{\top} \mu_{i, 0}-v_{i, M+1}^{\top} \mu_{i, M+1}$, the expression of the optimal cost follows as claimed.

\section{Optimal semi-state feedback control}

In this section we turn our attention to characterising the existence of a semistate feedback realisation of the LQ optimal control. That is,

$$
u_{i, j}=\left[\begin{array}{ll}
K_{i, j}^{h} & K_{i, j}^{v}
\end{array}\right]\left[\begin{array}{l}
h_{i, j} \\
v_{i, j}
\end{array}\right] .
$$

It is assumed that $H:=\operatorname{diag}\left\{H_{0}, H_{1}, \ldots, H_{M}\right\}$ and $V:=\operatorname{diag}\left\{V_{0}, V_{1}, \ldots, V_{N}\right\}$, so that the cost criterion (4) can be written as

$$
J=\sum_{j=0}^{M} h_{N+1, j}^{\top} H_{j} h_{N+1, j}+\sum_{i=0}^{N} v_{i, M+1}^{\top} V_{i} v_{i, M+1}+\sum_{(i, j) \in \mathcal{Q}}\left[h_{i, j}^{\top} v_{i, j}^{\top} u_{i, j}^{\top}\right] \Pi\left[\begin{array}{c}
h_{i, j} \\
v_{i, j} \\
u_{i, j}
\end{array}\right]
$$

where $\Pi=\left[\begin{array}{cc}Q & S \\ S^{\top} & R\end{array}\right]=\Pi^{\top} \geq 0$. Moreover, the north-east boundary constraints are discarded in this section, since it is not known how to handle such constraints in the presence of the structural constraint (29). Bearing this in mind, the semi-state feedback LQ optimal control problem can be stated as follows.

Problem 4.1 Find matrices $K_{i, j}^{h} \in \mathbb{R}^{m \times n_{h}}$ and $K_{i, j}^{v} \in \mathbb{R}^{m \times n_{v}}$, for $(i, j) \in$ $\mathcal{Q}$, so that the semi-state feedback controller defined in (29) minimises the performance index (30), subject to the constraints (1) and (3). 
A condition for the solvability of Problem 4.1 is provided in the statement of the following theorem, where a 2-D Riccati-like equation is introduced. When iteration of the Riccati-like equation is possible, the semi-state feedback matrices can be computed explicitly.

Theorem 4.1 Define $X_{N+1, j}=H_{j}$ for all $j \in[0, M]$ and $Y_{i, M+1}=V_{i}$ for all $i \in[0, N]$. If the Riccati difference equation

$$
\begin{aligned}
{\left[\begin{array}{cc}
X_{i, j} & 0 \\
0 & Y_{i, j}
\end{array}\right]=} & Q+A^{\top}\left[\begin{array}{cc}
X_{i+1, j} & 0 \\
0 & Y_{i, j+1}
\end{array}\right] A-\left(S+A^{\top}\left[\begin{array}{cc}
X_{i+1, j} & 0 \\
0 & Y_{i, j+1}
\end{array}\right] B\right) \\
& \cdot\left(R+B^{\top}\left[\begin{array}{cc}
X_{i+1, j} & 0 \\
0 & Y_{i, j+1}
\end{array}\right] B\right)^{\dagger}\left(S^{\top}+B^{\top}\left[\begin{array}{cc}
X_{i+1, j} & 0 \\
0 & Y_{i, j+1}
\end{array}\right] A\right)
\end{aligned}
$$

admits a corresponding solution for all $(i, j) \in \mathcal{Q}$, then:

(i) for all $(i, j) \in \mathcal{Q}$, the matrices $X_{i, j}$ and $Y_{i, j}$ satisfying (31) are symmetric and positive semi-definite;

(ii) the semi-state feedback controller

$$
\begin{aligned}
u_{i, j}= & -\left(R+B^{\top}\left[\begin{array}{cc}
X_{i+1, j} & 0 \\
0 & Y_{i, j+1}
\end{array}\right] B\right)^{\dagger}\left(S^{\top}+B^{\top}\left[\begin{array}{cc}
X_{i+1, j} & 0 \\
0 & Y_{i, j+1}
\end{array}\right] A\right)\left[\begin{array}{l}
h_{i, j} \\
v_{i, j}
\end{array}\right] \\
& +\left(I_{m}-\left(R+B^{\top}\left[\begin{array}{cc}
X_{i+1, j} & 0 \\
0 & Y_{i, j+1}
\end{array}\right] B\right)^{\dagger}\left(R+B^{\top}\left[\begin{array}{cc}
X_{i+1, j} & 0 \\
0 & Y_{i, j+1}
\end{array}\right] B\right)\right) \xi_{i, j}
\end{aligned}
$$

solves Problem 4.1, where for all $(i, j) \in \mathcal{Q}$ the vector $\xi_{i, j} \in \mathbb{R}^{m}$ is arbitrary; (iii) the optimal value of the performance index is

$$
J^{\star}=\sum_{j=0}^{M} h_{0, j}^{\top} X_{0, j} h_{0, j}+\sum_{i=0}^{N} v_{i, 0}^{\top} Y_{i, 0} v_{i, 0} .
$$

Proof: The proof below follows a line similar to the proof of the corresponding 1-D result in (Ferrante and Zampieri, 2003). First consider $(i)$. We proceed by induction, by first noticing that $X_{N+1, j}=H_{j}=H_{j}^{\top}=X_{N+1, j}^{\top} \geq 0$, for all $j \in[0, M]$, and $Y_{i, M+1}=V_{i}=V_{i}^{\top}=Y_{i, M+1}^{\top} \geq 0$, for all $i \in[0, N]$. Now consider an $(i, j) \in \mathcal{Q}$ and suppose that $X_{i+1, j}$ and $Y_{i, j+1}$ are both symmetric and positive semi-definite. Then the right-hand side of (31) is a symmetric matrix, so that $X_{i, j}$ and $Y_{i, j}$ are also symmetric. It therefore remains to show positive semi-definiteness. To this end, note that the matrix on the left-hand side of (31) coincides with the Schur pseudo-complement of the Popov matrix 


$$
\Pi+\left[\begin{array}{c}
A^{\top} \\
B^{\top}
\end{array}\right]\left[\begin{array}{cc}
X_{i+1, j} & 0 \\
0 & Y_{i, j+1}
\end{array}\right]\left[\begin{array}{ll}
A & B
\end{array}\right]
$$

which is clearly symmetric and positive semi-definite. In particular, with $\Theta_{i, j}:=$ $\left[\begin{array}{cc}X_{i+1, j} & 0 \\ 0 & Y_{i, j+1}\end{array}\right]$, for $(i, j) \in \mathcal{Q}$, it follows that

$$
\left[\begin{array}{cc}
Q+A^{\top} \Theta_{i, j} A & S+A^{\top} \Theta_{i, j} B \\
S^{\top}+B^{\top} \Theta_{i, j} A & R+B^{\top} \Theta_{i, j} B
\end{array}\right]=\Pi+\left[\begin{array}{c}
A^{\top} \\
B^{\top}
\end{array}\right] \Theta_{i, j}\left[\begin{array}{ll}
A & B
\end{array}\right] \geq 0 .
$$

and

$$
\begin{aligned}
& {\left[\begin{array}{cc}
X_{i, j} & 0 \\
0 & Y_{i, j}
\end{array}\right]=\left[I-\left(S+A^{\top} \Theta_{i, j} B\right)\left(R+B^{\top} \Theta_{i, j} B\right)^{\dagger}\right]\left[\begin{array}{cc}
Q+A^{\top} \Theta_{i, j} A & S+A^{\top} \Theta_{i, j} B \\
S^{\top}+B^{\top} \Theta_{i, j} A & R+B^{\top} \Theta_{i, j} B
\end{array}\right]} \\
& {\left[\begin{array}{c}
I \\
-\left(R+B^{\top} \Theta_{i, j} B\right)^{\dagger}\left(S^{\top}+B^{\top} \Theta_{i, j} A\right)
\end{array}\right] \geq 0,}
\end{aligned}
$$

since

$$
\left(R+B^{\top} \Theta_{i, j} B\right)\left(R+B^{\top} \Theta_{i, j} B\right)^{\dagger}\left(S+A^{\top} \Theta_{i, j} B\right)^{\top}=\left(S+A^{\top} \Theta_{i, j} B\right)^{\top} .
$$

This last identity follows from the inclusion

$$
\operatorname{ker}\left(S+A^{\top} \Theta_{i, j} B\right) \supseteq \operatorname{ker}\left(R+B^{\top} \Theta_{i, j} B\right) \quad \forall(i, j) \in \mathcal{Q}
$$

by which there exists a matrix $\Xi$ such that $\left(S+A^{\top} \Theta_{i, j} B\right)^{\top}=\left(R+B^{\top} \Theta_{i, j} B\right) \Xi$, and the inclusion itself holds because, by the positive semi-definiteness of the Popov matrix $\Pi$, there exists matrices $C_{i, j}$ and $D_{i, j}$, for $(i, j) \in \mathcal{Q}$, such that $S+A^{\top} \Theta_{i, j} B=C_{i, j}^{\top} D_{i, j}$ and $R+B^{\top} \Theta_{i, j} B=D_{i, j}^{\top} D_{i, j}$, so that $v \in \operatorname{ker}(R+$ $\left.B^{\top} \Theta_{i, j} B\right)$ implies $v \in \operatorname{ker} D_{i, j}$, and hence, $v \in \operatorname{ker} C_{i, j}^{\top} D_{i, j}=\operatorname{ker}\left(S+A^{\top} \Theta_{i, j} B\right)$.

Now consider $(i i)$. In view of $(1)$, the identity

$$
\sum_{(i, j) \in \mathcal{Q}} h_{i+1, j}^{\top} X_{i+1, j} h_{i+1, j}-h_{i, j}^{\top} X_{i, j} h_{i, j}=\sum_{j=0}^{M} h_{N+1, j}^{\top} X_{N+1, j} h_{N+1, j}-h_{0, j}^{\top} X_{0, j} h_{0, j}
$$

can be re-written as 


$$
\begin{gathered}
\sum_{(i, j) \in \mathcal{Q}}\left[\begin{array}{lll}
h_{i, j}^{\top} & v_{i, j}^{\top} & u_{i, j}^{\top}
\end{array}\right]\left[\begin{array}{ccc}
A_{1,1}^{\top} X_{i+1, j} A_{1,1}-X_{i, j} & A_{1,1}^{\top} X_{i, j} A_{1,2} & A_{1,1}^{\top} X_{i+1, j} B_{1} \\
A_{1,2}^{\top} X_{i+1, j} A_{1,1} & A_{1,2}^{\top} X_{i+1, j} A_{1,2} & A_{1,2}^{\top} X_{i+1, j} B_{1} \\
B_{1}^{\top} X_{i+1, j} A_{1,1} & B_{1}^{\top} X_{i+1, j} A_{1,2} & B_{1}^{\top} X_{i+1, j} B_{1}
\end{array}\right]\left[\begin{array}{c}
h_{i, j} \\
v_{i, j} \\
u_{i, j}
\end{array}\right] \\
-\sum_{j=0}^{M} h_{N+1, j}^{\top} X_{N+1, j} h_{N+1, j}+h_{0, j}^{\top} X_{0, j} h_{0, j}=0
\end{gathered}
$$

and similarly for the vertical semi-state,

$$
\sum_{(i, j) \in \mathcal{Q}} v_{i, j+1}^{\top} Y_{i, j+1} v_{i, j+1}-v_{i, j}^{\top} Y_{i, j} v_{i, j}=\sum_{i=0}^{N} v_{i, M+1}^{\top} Y_{i, M+1} v_{i, M+1}-v_{i, 0}^{\top} Y_{i, 0} v_{i, 0}
$$

leads to

$$
\begin{aligned}
\sum_{(i, j) \in \mathcal{Q}} & {\left[h_{i, j}^{\top} v_{i, j}^{\top} u_{i, j}^{\top}\right]\left[\begin{array}{ccc}
A_{2,1}^{\top} Y_{i, j+1} A_{2,1} & A_{2,1}^{\top} Y_{i, j+1} A_{2,2} & A_{2,1}^{\top} Y_{i, j+1} B_{2} \\
A_{2,2}^{\top} Y_{i, j+1} A_{2,1} & A_{2,2}^{\top} Y_{i, j+1} A_{2,2}-Y_{i, j} & A_{2,2}^{\top} Y_{i, j+1} B_{2} \\
B_{2}^{\top} Y_{i, j+1} A_{2,1} & B_{2}^{\top} Y_{i, j+1} A_{2,2} & B_{2}^{\top} Y_{i, j+1} B_{2}
\end{array}\right]\left[\begin{array}{c}
h_{i, j} \\
v_{i, j} \\
u_{i, j}
\end{array}\right] } \\
& -\sum_{i=0}^{N} v_{i, M+1}^{\top} Y_{i, M+1} v_{i, M+1}+h_{i, 0}^{\top} Y_{i, 0} v_{i, 0}=0 .
\end{aligned}
$$

Adding these zero-valued terms to the performance index we get

$$
J=\sum_{j=0}^{M} h_{0, j}^{\top} X_{0, j} h_{0, j}+\sum_{i=0}^{N} v_{i, 0}^{\top} Y_{i, 0} v_{i, 0}+\sum_{(i, j) \in \mathcal{Q}}\left[h_{i, j}^{\top} v_{i, j}^{\top} u_{i, j}^{\top}\right] \bar{\Pi}_{i, j}\left[\begin{array}{c}
h_{i, j} \\
v_{i, j} \\
u_{i, j}
\end{array}\right]
$$

where

$$
\begin{aligned}
& \bar{\Pi}_{i, j}:=\left[\begin{array}{c}
Q+\left[\begin{array}{c}
A_{1,1}^{\top} \\
A_{1,2}^{\top}
\end{array}\right] X_{i+1, j}\left[\begin{array}{ll}
A_{1,1} & A_{1,2}
\end{array}\right]+\left[\begin{array}{l}
A_{2,1}^{\top} \\
A_{2,2}^{\top}
\end{array}\right] Y_{i, j+1}\left[\begin{array}{ll}
A_{2,1} & A_{2,2}
\end{array}\right] \\
S^{\top}+B_{1}^{\top} X_{i+1, j}\left[\begin{array}{ll}
A_{1,1} & A_{1,2}
\end{array}\right]+B_{2}^{\top} Y_{i, j+1}\left[\begin{array}{ll}
A_{2,1} & A_{2,2}
\end{array}\right]
\end{array}\right. \\
& \left.\begin{array}{rl}
S+ & \left.\left[\begin{array}{l}
A_{1,1}^{\top} \\
A_{1,2}^{\top}
\end{array}\right] X_{i+1, j} B_{1}+\left[\begin{array}{l}
A_{2,1}^{\top} \\
A_{2,2}^{\top}
\end{array}\right] Y_{i, j+1} B_{2}\right]-\left[\begin{array}{cc}
X_{i, j} & 0 \\
0 & Y_{i, j}
\end{array}\right] \\
0 & \\
& R+B_{1}^{\top} X_{i+1, j} B_{1}+B_{2}^{\top} Y_{i, j+1} B_{2}
\end{array}\right]
\end{aligned}
$$

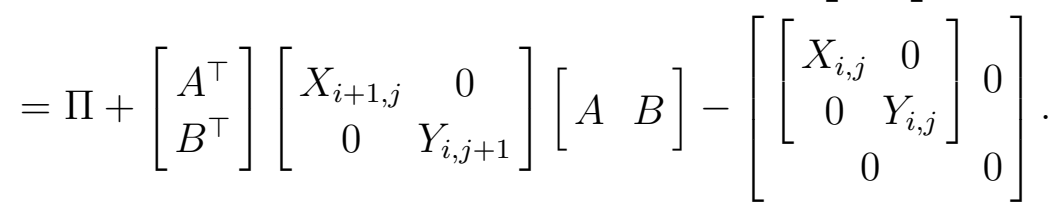


By using (31) we find

$$
\bar{\Pi}_{i, j}=\left[\begin{array}{cr}
\left(S+A^{\top} \Theta_{i, j} B\right)\left(R+B^{\top} \Theta_{i, j} B\right)^{\dagger}\left(S^{\top}+B^{\top} \Theta_{i, j} A\right) & S+A^{\top} \Theta_{i, j} B \\
S^{\top}+B^{\top} \Theta_{i, j} A & R+B^{\top} \Theta_{i, j} B
\end{array}\right]
$$

By using (33), it is found that $\bar{\Pi}_{i, j}$ can be written as

$$
\bar{\Pi}_{i, j}=\left[\begin{array}{c}
S+A^{\top} \Theta_{i, j} B \\
R+B^{\top} \Theta_{i, j} B
\end{array}\right]\left(R+B^{\top} \Theta_{i, j} B\right)^{\dagger}\left[S^{\top}+B^{\top} \Theta_{i, j} A R+B^{\top} \Theta_{i, j} B\right]
$$

As a result, we get

$$
\begin{aligned}
J= & \sum_{j=0}^{M} h_{0, j}^{\top} X_{0, j} h_{0, j}+\sum_{i=0}^{N} v_{i, 0}^{\top} Y_{i, 0} v_{i, 0}+\sum_{(i, j) \in \mathcal{Q}}\left[h_{i, j}^{\top} v_{i, j}^{\top} u_{i, j}^{\top}\right] \\
& \cdot\left[\begin{array}{c}
S+A^{\top} \Theta_{i, j} B \\
R+B^{\top} \Theta_{i, j} B
\end{array}\right]\left(R+B^{\top} \Theta_{i, j} B\right)^{\dagger}\left[S^{\top}+B^{\top} \Theta_{i, j} A R+B^{\top} \Theta_{i, j} B\right]\left[\begin{array}{c}
h_{i, j} \\
v_{i, j} \\
u_{i, j}
\end{array}\right] .
\end{aligned}
$$

Therefore, if a control input $u_{i, j}$ exists such that, for all $(i, j) \in \mathcal{Q}$,

$$
N_{i, j}\left(\left(S^{\top}+B^{\top} \Theta_{i, j} A\right)\left[\begin{array}{l}
h_{i, j} \\
v_{i, j}
\end{array}\right]+\left(R+B^{\top} \Theta_{i, j} B\right) u_{i, j}\right)=0
$$

where $N_{i, j}^{\top} N_{i, j}=\left(R+B^{\top} \Theta_{i, j} B\right)^{\dagger}$, then the performance index (4) is minimised, and its optimal value is

$$
J^{\star}=\sum_{j=0}^{M} h_{0, j}^{\top} X_{0, j} h_{0, j}+\sum_{i=0}^{N} v_{i, 0}^{\top} Y_{i, 0} v_{i, 0} .
$$

We now show that condition (36) is equivalent to

$$
\left(S^{\top}+B^{\top} \Theta_{i, j} A\right)\left[\begin{array}{l}
h_{i, j} \\
v_{i, j}
\end{array}\right]+\left(R+B^{\top} \Theta_{i, j} B\right) u_{i, j}=0
$$

The fact that (37) implies (36) is trivial. Concerning the opposite implication, we premultiply (36) by $\left(R+B^{\top} \Theta_{i, j} B\right) N_{i, j}^{\top}$, so that the quantity 


$$
\left(R+B^{\top} \Theta_{i, j} B\right)\left(R+B^{\top} \Theta_{i, j} B\right)^{\dagger}\left(\left(S^{\top}+B^{\top} \Theta_{i, j} A\right)\left[\begin{array}{c}
h_{i, j} \\
v_{i, j}
\end{array}\right]+\left(R+B^{\top} \Theta_{i, j} B\right) u_{i, j}\right)
$$

is zero. By using (33) the former can be simplified to

$$
\left(S^{\top}+B^{\top} \Theta_{i, j} A\right)\left[\begin{array}{c}
h_{i, j} \\
v_{i, j}
\end{array}\right]+\left(R+B^{\top} \Theta_{i, j} B\right) u_{i, j}=0
$$

In view of (34), the solutions of (38) are parameterised as

$$
u_{i, j}=-\left(R+B^{\top} \Theta_{i, j} B\right)^{\dagger}\left(S^{\top}+B^{\top} \Theta_{i, j} A\right)\left[\begin{array}{l}
h_{i, j} \\
v_{i, j}
\end{array}\right]+\bar{u}_{i, j}
$$

where for all $(i, j) \in \mathcal{Q}$ the vector $\bar{u}_{i, j}$ lies in the null-space of $R+B^{\top} \Theta_{i, j} B$. However, since

$$
\operatorname{ker}\left(R+B^{\top} \Theta_{i, j} B\right)=\operatorname{im}\left(I_{m}-\left(R+B^{\top} \Theta_{i, j} B\right)\left(R+B^{\top} \Theta_{i, j} B\right)^{\dagger}\right),
$$

it follows that the set of solutions of (38) can be alternatively parametrised as shown in (32).

Remark 4.1 Theorem 4.1 provides a sufficient condition for the existence of a semi-state feedback regulator that minimises the performance index (30). For (31) and the boundary condition to admit a solution for all $(i, j) \in \mathcal{Q}$, it is necessary for the right-hand side to preserve the block diagonal structure; i.e. to be consistent with the left-hand side. Notice that (31) can be either iterated by row or by column in this case. The first step consists in using (31) with $i=N$ and $j=M$, so as to assign $X_{N, M}$ and $Y_{N, M}$. Then, one may proceed by rows, computing $X_{k, M}$ and $Y_{k, M}$ for all $k$ backwards from $N-1$ to 0 . In this way, all the matrices $X_{i, j}$ and $Y_{i, j}$ are assigned in the $M$-th row. Then, starting from $X_{N-1, M-1}$ and $Y_{N-1, M-1}$, all the matrices in the $(M-1)$-th row can be computed. Alternatively, one may proceed by columns, computing $X_{N, h}$ and $Y_{N, h}$ for all $h$ from $M-1$ to 0 , and so on. Similarly, the matrices can be computed in a zig-zag fashion.

\section{$5 \quad$ Concluding remarks}

In this paper we study finite-extent open-loop LQ optimal control, and a similar problem subject to the structural constraint of a semi-state feedback, for 2-D Roesser models. We provide necessary and sufficient conditions for 
optimality and explore situations in which it is possible to explitly compute optimal open-loop control laws. In the second part of the paper, a 2-D Riccatilike recursion is introduced to characterise, in a sufficient sense, a solution in the form of a semi-state feedback. This recursion is required to preserve a particular structure. Characterising when this is the case, directly in terms of the problem data, appears to be difficult and clearly constitutes an important direction for further investigation.

\section{References}

R. Roesser, "A discrete state-space model for linear image processing," IEEE Transactions on Automatic Control, vol. AC-20, no. 1, pp. 1-10, 1975.

E. Fornasini and G. Marchesini, "Doubly-Indexed Dynamical Systems: State-Space Models and Structural Properties," Mathematical System Theory, vol. 12, pp. 59-72, 1978.

M. Bisiacco and E. Fornasini, "Optimal control of two-dimensional systems," SIAM Journal of Control and Optimization, vol. 28, pp. 582-601, 1990.

M. Bisiacco, "New Results in 2D Optimal Control Theory," Multidimensional Systems and Signal Processing, vol. 6, pp. 189-222, 1995.

A. Ferrante and L. Ntogramatzidis, "Employing the algebraic Riccati equation for a parametrization of the solutions of the finite-horizon LQ problem: the discrete-time case". Systems \& Control Letters, 54:693-703, 2005 .

A. Ferrante and S. Zampieri, "A simple derivation of singular linear-quadratic optimal controller for discrete-time systems," unpublished, October 2003.

T. Kaczorek, Two-Dimensional Linear Systems, Springer-Verlag, 1985.

C. Li and M. Fadali, "Optimal control of 2-D systems," IEEE Transactions on Automatic Control, vol. AC-36, no. 2, pp. 223-228, February 1991.

O. Mangasarian, "Sufficient conditions for the optimal control of nonlinear systems," SIAM Journal on Control, vol. 4, no. 1, pp. 139-152, 1966.

T. Kaczorek and J. Klamka, "Minimum energy control of 2-D linear systems with variable coefficients," International Journal of Control, vol. 44, no. 3, pp. 645-650, 1986. 